\title{
Earn-outs als Weg zur Praxisübergabe
}

\author{
Patrick Stacha,b, Ramon Bühler
}

${ }^{a}$ Dr.; ${ }^{b}$ Rechtsanwalt und Notar, Stach Rechtsanwälte AG Zürich/St. Gallen

\begin{abstract}
Nicht selten scheitern Praxisnachfolgen an unterschiedlichen Preisvorstellungen oder an der Finanzierung des Kaufpreises. In solchen Situationen sollte die Earnout-Methode in Betracht gezogen werden. Mit ihr wird ein Teil des Kaufpreises aufgeschoben und variabel vom zukünftigen Erfolg abhängig gemacht. Earn-out-Vereinbarungen gelten daher als sinnvolle Alternative bei Praxisverkäufen. Die vertragliche Ausgestaltung ist jedoch nicht zu unterschätzen und sollte auf jeden Fall unter Beizug einer Fachperson erfolgen, damit einer erfolgreichen Praxisübernahme nichts mehr im Weg steht.
\end{abstract}

\section{Der Schritt in die Pension}

Ein grosser Teil der Baby-Boomer-Generation steht kurz vor dem Pensionsalter. Die wenigen noch bevorstehenden Arbeitsjahre erscheinen kurz im Vergleich zum ganzen Berufsleben, in dem man erfolgreich eine eigene Praxis aufgebaut und gepflegt hat. Doch in genau diesen Jahren ist es wichtig, dass frühzeitig an eine Frage gedacht wird: Wie übergebe ich meine Praxis an einen Nachfolger ${ }^{1}$ ?

Es gibt etliche Wege, den Verkauf einer Praxis vertraglich auszugestalten. Eine Möglichkeit ist die Übergabe der gesamten Praxis mit allen Einrichtungen, Verträgen und Mitarbeitenden mittels Einmalabfindung. Eine andere Möglichkeit bietet sich in einem Earn-out. Ein Earn-out ist gerade deshalb sehr

\section{Résumé}

II n'est pas rare que la reprise d'un cabinet échoue en raison de divergences de vues sur les prix ou du financement du prix d'achat. Dans de tels cas, la méthode de l'earn-out devrait être envisagée. Celle-ci permet de reporter le versement d'une partie du prix d'achat et de l'indexer sur les résultats futurs. Les accords d'earn-out sont donc considérés comme des alternatives judicieuses lors de ventes de cabinets. L'étape de la rédaction du contrat ne doit cependant pas être sous-estimée et il faut impérativement faire appel à un spécialiste pour que plus rien ne s'oppose à une reprise de cabinet réussie.

vielversprechend, weil die Übernahme bzw. Übergabe einer gut funktionierenden Arztpraxis vielfach am Preis und dessen Finanzierung scheitert. Mit einer sogenannten Earn-out-Klausel lassen sich Praxisübernahmen bzw. -übergaben im Gegensatz zum Verkauf mittels Einmalzahlung für sämtliche Beteiligten zu bestmöglichen Konditionen vollziehen.

Ein potentieller Nachfolger geht bei einer Praxisübernahme immer ein wirtschaftliches Risiko ein. Nicht

Ein potentieller Nachfolger geht bei einer Praxisübernahme immer ein wirtschaftliches Risiko ein.

nur weil er seinen beruflichen Mittelpunkt in ein neues Umfeld rückt, sondern auch weil er oftmals eine nicht unbeträchtliche Summe Geld in eine Praxis investiert, deren Wert nicht präzise beziffert werden kann. Während eine Übergabe mittels Einmalabfindung eine Praxisbewertung bedingt, die insbesondere in Bezug auf den Patientenstamm nur sehr schwierig zu bewerkstelligen ist, wird bei einem Earn-out der Praxiswert nicht als feststehende Grösse eruiert. Vielmehr wird grundsätzlich sowohl ein Fixpreis für den objektiv bewertbaren Substanzwert inkl. stiller Reserven als auch eine gestaffelte Erfolgsbeteiligung vereinbart. Im Grunde genommen kann der komplette Kaufpreis jedoch variabel ausgestaltet werden, und sowohl positive (Zusatzzahlungen) als auch negative Earn-outKlauseln (Kaufpreisrückzahlungen) sind denkbar. 
Ferner hat der übergebende Arzt durch den Earn-outAnsatz ein Interesse daran, eine gut funktionierende Praxis zu übergeben und mit vollem Engagement bis zur letzten Sekunde weiterzuarbeiten, zumal er weiterhin am Erfolg der von ihm aufgebauten Praxis partizipiert. Der Übernehmer hingegen weiss, dass er gerade für den nur sehr ungenau zu beziffernden Patientenstamm keine überhöhte Zahlung leistet. Durch eine Earn-out-Vereinbarung minimiert sich das Risiko also für sämtliche Beteiligten: Der Übergeber kann sichergehen, dass die Finanzierung des Verkaufspreises mit genügend liquiden Mitteln gedeckt sein wird und erhält bei einer erfolgreichen Übergabe wesentlich mehr als bei einer reinen Goodwillzahlung für den Patientenstamm. Demgegenüber kauft der Übernehmer keine Blackbox und muss grundsätzlich nur das bezahlen, was durch die Weiterführung der Praxis erwirtschaftet wird.

\section{Vorteile einer Zusammenarbeit}

Vielen Ärzten fällt es schwer loszulassen, ihr Lebenswerk von einem Tag auf den anderen in fremde Hände zu geben. Bei einer klassischen Praxisübergabe verbleibt der Seniorpartner daher nach Vertragsabschluss noch für eine vertraglich bestimmte Zeit in "seiner» Praxis. Dies ermöglicht einen sukzessiven Austritt aus der Praxis und somit einen fliessenden Übergang in die Pension. Durch die weitere Mitarbeit im Unternehmen kann der Übergeber zudem positiv auf das Geschäftsgeschehen einwirken und damit dazu beitragen, dass die Praxis nicht nur weiterhin floriert, sondern auch seine eigene Abfindung maximieren.

\section{Vielfach scheitert die Übergabe bzw. Über- nahme an allfälligen Kollaborationsschwierig- keiten der beteiligten Ärzte.}

In einer möglichen Zusammenarbeit zwischen übergebendem und übernehmendem Arzt liegt aber ein weiterer Stolperstein. Vielfach scheitert die Übergabe bzw. Übernahme nämlich nicht an den Preisvorstellungen der Beteiligten, sondern an allfälligen Kollaborationsschwierigkeiten der beteiligten Ärzte. Diesem Fallstrick wird mit der Earn-out-Klausel ebenfalls entgegengewirkt: Beide Parteien haben ein reges Interesse daran, dass die Praxis weiterhin erfolgreich wirtschaftet. Gerade im Dienstleistungssektor steht der menschliche Kontakt im Vordergrund, und somit gilt die Beziehung zwischen einem Arzt und seinen Patienten als entscheidender Faktor für den Erfolg einer Praxis. Durch die, wenn auch nur temporäre, Zusammenarbeit beider Vertragsparteien kann der Übergeber die
Patienten mit seinem Nachfolger vertraut machen und den Beziehungsaufbau erleichtern. Dadurch kann der Wert des Patientenstamms bestmöglich erhalten werden, wovon beide Vertragsparteien gleichermassen profitieren. Aber auch die Patienten gewinnen. Sie werden nicht vor vollendete Tatsachen gestellt, sondern

\section{Beide Parteien haben ein reges Interesse} daran, dass die Praxis weiterhin erfolgreich wirtschaftet.

können langsam eine neue Vertrauensbeziehung aufbauen, wodurch sie erhebliche Wechselkosten verhindern können. Somit stellt diese Fallkonstellation eine Win-win-win-Situation dar.

\section{Mögliche Steuerfolgen beachten}

Ferner kommen steuerliche Aspekte dazu. Das Ausmass der steuerlichen Vorteile und Risiken variiert je nach individueller Vertragsausgestaltung, weshalb in diesem Artikel nicht vertieft darauf eingegangen wird. Ein guter Berater wird diesen Faktor aber nicht nur erkennen, sondern auch in der Lage sein, die steuerlichen Folgen zuverlässig abzuschätzen. Anfang der Steuerdiskussion wird die rechtliche Ausgestaltung der Arztpraxis und die Frage sein, ob eine Umstrukturierung mittels Umwandlung der Praxis in eine Aktiengesellschaft notwendig ist, um die Praxis übergabe steuer- und sozialversicherungsrechtlich sowie arbeitsrechtlich optimal in die Wege leiten zu können.

\section{Vertragsausgestaltung als Grundstein des Erfolgs}

Damit die Praxisübergabe mit Earn-out erfolgreich verläuft und sich nicht als Quelle diverser Streitigkeiten entpuppt, gilt es einige wichtige Punkte zu beachten. Denn selbstredend sind auch Earn-outs nicht frei von Nachteilen. So bedingen sie u.a. eine komplexere Vertragsausgestaltung. Gerade die Ausgestaltung der Earn-out-Formel sollte keinen Raum für Interpretationen bieten und von einem erfahrenen Wirtschaftsjuristen aufgesetzt werden. Sind die massgeblichen Kennzahlen nämlich nicht präzis bestimmt oder einem zu grossen buchhalterischen Spielraum (z.B. in Bezug auf die Höhe von Abschreibungen) ausgesetzt oder anderweitig manipulierbar, sind juristische Auseinandersetzungen vorprogrammiert. Neben einer sorgfältigen Wahl der geeigneten Kennzahlen ist zudem die Ernennung einer unabhängigen Prüfstelle rat- 
sam, die bei Uneinigkeiten als praxiserprobter Mediator schlichtend zur Seite steht.

Ferner gilt es Kompetenzstreitigkeiten vertraglich vorzubeugen. Arbeitet der verkaufende Arzt noch weiter in der Praxis mit, so wird er aufgrund seiner vielen Jahre in der Praxis weiterhin eine zentrale Ansprech-, Führungs- und Vertrauensperson, insbesondere in Bezug auf Mitarbeitende und Patienten, sein. Dabei stellen sich Fragen der Kompetenzregelung. Soll der Seniorpartner noch eine Weile die Geschicke der Praxis steuern können, oder kommt ihm von Anfang an eher eine Art Patenrolle ohne Mitspracherecht zu? Wer hat in welcher Situation das letzte Wort? Mit Hilfe einer klar strukturierten vertraglich vereinbarten Kompetenzordnung kann sichergestellt werden, dass Kompetenzstreitigkeiten gar nicht erst aufkommen. Und für den Fall, dass dennoch Spannungen auftreten sollten, sieht eine umfassende Übergabevereinbarung Schlichtungsmechanismen oder, als ultima ratio, beidseitige Vertragsrücktrittsklauseln oder Auskaufsmöglichkeiten vor.

Genauso wichtig wie eine geregelte Kompetenzordnung sind die Modalitäten der Earn-out-Vereinbarung. Es sollte klar festgehalten werden, über welchen Zeitraum Earn-out-Zahlungen geschuldet sowie wann diese zu entrichten sind. Sollen jährliche Zahlungen vereinbart werden, oder soll eine kumulierte Zahlung am Ende der Earn-out-Periode erfolgen? Sind die jährlichen Beteiligungen im Falle einer aufgeschobenen

\section{Genauso wichtig wie eine geregelte Kompe- tenzordnung sind die Modalitäten der Earn-out- Vereinbarung.}

Zahlung zu verzinsen? Andererseits bedeutet die Festlegung der Dauer auch die Vereinbarung eines fixierten Enddatums, an dem die allfällige Zusammenarbeit endet und der Seniorpartner aus der Arztpraxis zurücktritt.

Möchte der übergebende Arzt nicht mehr in der Praxis mitwirken, so gilt es sicherzustellen, dass Einsichtsrechte in die Geschäftsbücher für die Überprüfung der Berechnung des Earn-outs dennoch vereinbart werden. Weiter kann es sinnvoll sein, dass dem Übergeber ein Recht auf regelmässiges Reporting und Teilnahme an wichtigen Sitzungen gewährt oder eine notwendige Zustimmung beider Parteien bei gewissen Geschäftsvorfällen vereinbart wird. Auch hier empfiehlt sich die

Ein Experte kann den Earn-out-Vertrag optimal auf die Bedürfnisse der Vertragsparteien zuschneiden.

Ernennung einer unabhängigen Prüf- und Schlichtungsstelle. Nebst Einsichts-, Informations- und Mitspracherechten können aber auch Vereinbarungen über Mindestbeträge sowie die Sicherstellung von Zahlungen der Erfolgsanteile Thema sein.

Den genannten Problemfeldern und Stolpersteinen kann ein Experte entgegenwirken, indem er den Earnout-Vertrag optimal auf die Bedürfnisse der beiden Vertragsparteien zuschneidet und mögliche Fallstricke schon im Vorfeld anspricht. Im Übrigen wird auf die Publikation von Dr. Patrick Stach in der Schweizerischen Ärztezeitung [1] verwiesen, die weitere Stolpersteine bei einer Praxisübernahme anspricht.

\section{Fazit}

Zusammenfassend ist festzustellen, dass Earn-outs eine vielversprechende (neue) Möglichkeit zur Praxisübergabe bieten. Die Vorteile liegen dabei insbesondere in der allenfalls gewünschten Möglichkeit eines schrittweisen Eintritts in die Pension, in der einfacheren und mit weniger Risiken behafteten Finanzierung sowie in der Ausnützung möglicher Steuervorteile. Die Formulierung von auf den Einzelfall zugeschnittenen Earn-out-Klauseln jedoch ist nicht einfach und erfordert einerseits ein tiefgründiges Verständnis der Branche und andererseits jahrelange Erfahrung in der Ausgestaltung diverser Deals. Bei frühzeitiger Involvierung kann ein Experte nicht nur dabei behilflich sein, einen passenden Vertrag aufzusetzen, sondern den Parteien auch bei der Erreichung ihrer gemeinsamen Ziele beratend zur Seite stehen.

\footnotetext{
Literatur

1 Stach P, Teichmann F. Stolpersteine bei der Praxisübernahme. Schweiz Arzteztg. 2016:97(24):898-900.
} 